\title{
ALCUNI CENNI SUGLI ITALIANISMI IN SLOVENO
}

\section{PREMESSA}

Come è tipico delle aree di confine tra due o più lingue, anche nel caso dello sloveno e dell'italiano si possono osservare diversi fenomeni dovuti al contatto linguistico, uno dei temi di ricerca preferiti dal nostro Festeggiato. Gli scambi interlinguistici lessicali, morfosintattici e altri - sono infatti tra le testimonianze più profonde della convivenza tra due o più lingue, specialmente nelle situazioni caratterizzate da una antica e continua presenza di contatto. Parlando dell'italiano e dello sloveno, e limitandoci ai lessemi italiani in sloveno, tralasciando quindi gli scambi nella direzione opposta, cioè gli elementi sloveni entrati in italiano - comunque meno numerosi e per lo più limitati alle varietà dialettali dell'area di confine - possiamo osservare che si tratta di prestiti assai numerosi e semanticamente molto eterogenei. Ricordiamo che gli italianismi presenti nello sloveno moderno rappresentano il secondo strato nell'insieme dei prestiti linguistici entrati in questa lingua dalle vicine parlate romanze nei diversi periodi di contatto. Infatti, prima della differenziazione linguistica dalla quale hanno avuto origine l'italiano e lo sloveno, nella parlata slava che costituiva l'antenato dello sloveno moderno erano entrati diversi lessemi di origine romanza a partire dalla seconda metà del VI secolo, periodo in cui le popolazioni slave si sarebbero insediate sul territorio delle Alpi Orientali dove vennero a contatto con i vicini romanzi. Tali lessemi costituiscono il primo strato del contatto e sono stati studiati in modo approfondito da diversi romanisti. Tra i contributi più importanti si vogliono ricordare gli studi, ormai classici, di Fran Šturm $(1927,1928)$ e quelli recenti di Agata Šega $(1998,2007,2008)$. Rientrano nel gruppo dei più antichi elementi romanzi lessemi come pogača (FOCACEA 'focaccia'), hlača (CALCEA 'calza', pl. hlače 'pantaloni'), kudati, dial. (COGITARE 'pensare') e križ (CRUCE(M) 'croce'). È inoltre presente in sloveno qualche altro romanismo ancora più antico, come češnja 'ciliegia', dal latino volgare CERESIA, entrato nello slavo già prima della migrazione di una parte dei parlanti slavi sul territorio summenzionato.

In questo breve contributo, volto ad offrire alcuni cenni introduttivi al tema degli italianismi in sloveno, ci soffermeremo piuttosto su elementi meno antichi: quelli entrati in sloveno dall'italiano, o spesso dai suoi dialetti perlopiù veneti incluso il triestino, oppure dal friulano. Saranno presi in considerazione solo prestiti linguistici, cioè lessemi di base italiana, mentre saranno esclusi, tranne qualche caso isolato, $\mathrm{i}$ calchi, che sono in parte già stati studiati (Skubic 2000).

\footnotetext{
* Indirizzo dell'autrice: Filozofska fakulteta, Oddelek za romanske jezike in književnosti, Aškerčeva 2, 1000 Ljubljana, Slovenia. Email: Martina.Ozbot@guest.arnes.si
} 


\section{LA SITUAZIONE LESSICOGRAFICA E IL METODO PER LA RACCOLTA DEGLI ITALIANISMI}

A questo punto ci si potrebbe chiedere quali possano essere i motivi per la mancanza di indagini sistematiche sui prestiti italiani, la cui origine risulta peraltro spesso ben riconoscibile a differenza dei calchi ove la matrice straniera è mascherata sotto il materiale linguistico "nativo». In larga misura la spiegazione va cercata nel fatto che fino a un decennio fa non c'era a disposizione un completo dizionario etimologico della lingua slovena, strumento indispensabile per lo studio dei prestiti linguistici. Nel 1997 è stato pubblicato il Dizionario etimologico sloveno (SES) di Marko Snoj, seguito, nel 2003, dalla seconda edizione ampliata e riveduta. In realtà, il Dizionario etimologico della lingua slovena (ESSJ) di France Bezlaj, opera magistrale in cinque volumi, ha iniziato ad essere pubblicato sin dal 1976, ma per l'uscita dell'ultimo volume si è dovuto aspettare fino al 2007. Per di più, lo scopo di Bezlaj era quello di esaminare scrupolosamente anche il materiale dialettale, rispetto al quale vari elementi della lingua standard venivamo spesso relegati in una posizione subalterna e pertanto esclusi dalla trattazione. Il dizionario di Snoj, invece, pur cercando di prendere in esame in primo luogo gli elementi dello sloveno moderno nella sua varietà standard, rivela un'impostazione abbastanza ampia da permettere anche l'inclusione del materiale d'uso regionale, nonché di quello dei diversi sottocodici e registri.

Poiché nella ricerca delineata in questo contributo ci si è voluti incentrare soprattutto sugli italianismi nella lingua standard, è stato $S E S$ a servire come fonte principale e ad essere esaminato lemma per lemma, mentre ESSJ è stato consultato solo nell'analisi di alcuni lessemi. In base a SES è stata di seguito compilata una lista di circa 450 italianismi presenti nella lingua slovena. Sono stati individuati inoltre alcuni elementi non inclusi né in SES né in ESSJ, ma esistenti nello sloveno contemporaneo e normalmente anche registrati dal Dizionario dello sloveno standard (SSKJ) o dal Lessico della lingua slovena (BSJ). Tra di essi si possono incontrare lessemi come čao, čau, čav, ciao 'ciao', pinjola 'pinolo'; škarpina/škarpena 'scorfano'; njok 'gnocco' ecc. Si può quindi stimare che il numero complessivo degli italianismi in sloveno, ad esclusione di elementi di diffusione prettamente locale, si aggiri intorno a 500.

\section{DEFINIZIONE DELL'ITALIANISMO E PROBLEMI DI DETERMINAZIONE DELLA BASE}

Nell'ambito della presente ricerca vengono considerati come italianismi tutti quei lessemi che sono entrati in sloveno dall'italiano o dai suoi dialetti, particolarmente dal triestino o da altre parlate di tipo veneto, sia direttamente oppure tramite una terza lingua, di solito il tedesco, il croato o il francese. In non pochi casi risulta impossibile constatare con certezza se si tratta di un italianismo o meno, in quanto la possibile origine di diversi lessemi potrebbe essere anche il friulano, che è stato una fonte importante dei prestiti romanzi in sloveno. Così, per esempio, nel caso del lessema podgana 'ratto (Rattus)' (coll. it. pantegana), potremmo supporre una base simile a quella del veneto pantegàna, pategàna o del friulano pantiàne, dal latino volgare 
*PONTICĀNA, il cui esito sloveno è dovuto sia all'assimilazione $t g \rightarrow d g$ sia all'influsso del prefisso sloveno pod-('sotto') (SES 532; Šturm 1927: 57). In modo analogo, anche per žoga ('palla') si potrebbe supporre una base vicina al veneto zogo, ziogo ('gioco'), zogàr, zogàr 'giocare' oppure al friulano zûc 'gioco', zuiâ, zuâ 'giocare' (SES 873).

\section{PRESTITI INDIRETTI}

Molto frequentemente, per la terminologia specialistica o per quella generale, l'intermediario è stata la lingua tedesca. Diversamente dal francese, il tedesco ha avuto un ruolo di mediazione importante probabilmente dai tempi remoti, almeno dal XI secolo in poi, quando gli sloveni furono sottoposti a un forte e costante influsso di questa lingua (Šega 2008: 82). Tra i numerosi casi appartenenti ai più svariati campi semantici si possono citare improvizirati 'improvvisare' (ted. improvisieren), bankrot, $m$ 'bancarotta' (ted. Bankrott, $m$ ), kapuca, $f$ 'cappuccio' (ted. Kapuze, f), mandolina, $f$ 'mandolino' (ted. Mandoline, $f$ ), karfjola 'cavolfiore' (ted. Karfiol, $m$ ); va notato che nei primi tre casi il tedesco ha influito anche sul genere dei prestiti. In diversi casi ha avuto un ruolo importante la varietà austriaca, come è dimostrato da lessemi come piškot 'biscotto', ted. austr. Biscotte, Piskotte; marela coll. 'ombrello', ted. austr. Amrel; feferon 'peperoncino', ted. austr. Pfefferone ecc. Talvolta gli italianismi sono giunti in sloveno attraverso il francese (es. ambasada 'ambasciata', fr. ambassade; fajansa 'faenza', fr. faïence), ma in realtà anche in questi casi non si può escludere con certezza la mediazione del tedesco. Il croato è stata lingua mediatrice, tra l'altro, nei casi di kolajna 'medaglia' (da collana, cr. medalja) e mornar 'marinaio' (dal veneto marinèr, cfr. it. antico marinario, cr. mornar; forse anche sotto l'influsso dello sloveno morje 'mare'; Sturm 1927: 72; SES 415), nonché nei casi di diversi ittionimi (škarpina/škarpena 'scorfano', cr. škarpina; sipa 'seppia', cr. sipa). Sia menzionato, nello stesso campo semantico, pure il calco oslič 'asinello (Merluccius vulgaris)', anch'esso entrato in sloveno attraverso il croato (oslić; cfr. Bezlaj 1959/60: 171). In qualche caso isolato i lessemi italiani sono giunti per la mediazione di altre lingue come ad esempio l'ungherese (es. pagat 'bagatto', ungh. pagát, dall'italiano bagatto), anche se pure qui non si potrebbe escludere l'influsso tedesco (cfr. SES 486).

Va aggiunto che elementi italiani sono presenti in numerosi altri prestiti, ma poiché il legame con l'italiano risulta piuttosto indiretto, nella nostra ricerca essi non sono stati considerati come italianismi. Lessemi di questo tipo sono riskirati ('rischiare', dal ted. riskieren, preso a prestito a sua volta dal fr. risquer, derivato da risque, dal ital. ant. risco), kalk ('calco linguistico' attraverso il ted. Kalk dal fr. calque 'copia, riproduzione', dal ital. calco 'impronta'), larifari 'ciancia, ciarla' (dal ted. Larifari, composto dai nomi di note musicali).

Si possono riscontrare anche dei casi in cui l'italiano stesso ha svolto il ruolo di mediazione: ad esempio, la parola livreja 'livrea' sarebbe entrata in sloveno dal francese livrée attraverso l'italiano livrea. Nella discussione sui prestiti indiretti è inoltre degno di menzione il lessema prošek 'prosecco', che attraverso l'italiano ha «fatto ritorno» in sloveno: presumibilmente si tratta di un toponimo di origine 
slovena (Prosek, nei dintorni di Trieste) che ha dato il nome al vino prosecco, poi rientrato in sloveno come italianismo.

\section{VALUTAZIONE D'INSIEME DEGLI ITALIANISMI}

Tra gli italianismi individuati nella lingua slovena ci sono differenze importanti relativamente alla frequenza d'uso, alla diffusione diatopica, al registro, al periodo in cui sono entrati in sloveno, ai campi semantici, al lievello di adattamento e ad altri criteri.

\section{V.1 Gli italianismi nel dialetto e in sloveno standard}

Va precisato che gli italianismi sono particolarmente frequenti nei dialetti sloveni occidentali, cioè nella zona di confine con l'Italia, dove lessemi di origine italiana riguardano i più svariati campi semantici. Dei numerosi italianismi dialettali - di diversa diffusione geografica e di diversa vitalità - annoveriamo solo una minima parte: šagra 'sagra', škartoc 'cartoccio', betula 'bettola', plenir 'paniere' (dal veneziano o triestno pianer, forse sotto l'influsso dello sloveno pleter 'cesto di vimini', plesti 'lavorare a maglia', v. Šturm 1927: 72), raca 'razza' ecc. Supponiamo che attraverso tali dialetti alcuni italianismi siano entrati anche nello sloveno standard (mineštra 'minestra', bakalá 'baccalà', melancana 'melanzana', cuketa 'zucchino'), dove numerosi italianismi esistono in parallelo con altri lessemi sinonimici dai quali differiscono talvolta per il registro d'uso (es. špargelj / beluš 'asparago', con il primo dei due lessemi appartenente al registro familiare; soldat / vojak 'soldato', dove il primo dei due lessemi è percepito come arcaico) o per specifici tratti semantici (per esempio, i verbi izolirati / osamiti 'isolare' sono in genere sinonimi, però non risultano reciprocamente sostituibili in tutti i contesti).

\section{2 La cronologia dell'afflusso degli italianismi}

Per quel che riguarda la datazione dell'entrata dei singoli italianismi in sloveno, essa è spesso impossibile da stabilire con precisione soddisfacente. SES fornisce l'informazione sul secolo in cui un dato lessema, per quanto ci è dato di sapere, risulta registrato per la prima volta in un testo sloveno. Specialmente nel caso degli italianismi antichi può trattarsi di un elemento presente nella lingua già da tempo, ma non usato nei testi scritti, o perlomeno non in quei testi scritti che abbiamo a disposizione (Snoj 2003: IV). L'italianismo più antico tra quelli compresi in SES sarebbe dukat 'ducato', la prima occorrenza del quale risalirebbe al XV secolo. Alcune decine di italianismi risalgono ai secoli XVI-XVIII, mentre la stragrande maggioranza è entrata in sloveno nei secoli XIX e XX. È interessante che, a giudicare da $S E S$, risalgono al XVI secolo circa 50 italianismi - tra questi troviamo alcuni nomi di piante, in particolare quelle commestibili (artičoka 'carciofo', špargelj 'asparago', čebula 'cipolla'), nonché termini associati alla vita militare e pubblica (bandero/bandera 'bandiera', galeja 'galea') e singoli lessemi appartenenti ad altri campi semantici (libra 'libbra', oštarija 'osteria', rufijan 'ruffiano'), mentre nel secolo successivo l'afflusso di lessemi italiani si è dimezzato (tra i pochi casi si possono 
citare soldat 'soldato', lazaret 'lazzaretto', cekin 'zecchino'). È probabile che il calo sia dovuto a un generale impoverimento della cultura avvenuto con la Controriforma, rispetto alla fioritura della lingua e della letteratura slovena promossa dagli scrittori protestanti attivi nella seconda metà del XVI secolo. Dal XVIII secolo si può osservare una costante crescita dell'afflusso degli italianismi in sloveno (intorno a 75 lessemi nel XVIII secolo, 120 nel XIX secolo e 180 nel XX secolo) che riflette, tra l'altro, il progressivo moltiplicarsi del materiale testuale scritto.

Oggi alcuni italianismi risultano obsoleti nello sloveno standard e allo stesso tempo caratteristici del linguaggio letterario (peza 'peso', briga 'preoccupazione') o del registro familiare (dac 'dazio'). Anche tra gli altri italianismi, che sono senza connotazioni arcaiche, troviamo degli elementi tipici del registro familiare (borša 'borsa', fešta 'festa', kapirati 'capire', giro 'giro (breve passeggiata, breve viaggio)', mulo 'ragazzo', mula 'ragazza', mularija 'ragazzaglia', 'ragazzi'). In qualche caso le basi italiane dalle quali derivano gli italianismi sloveni hanno perso vitalità nell'italiano moderno (pomaranča 'arancia' da pomarancia, goljuf 'imbroglione' da gaglioffo, romar 'pellegrino' da romero).

\section{3 I principali campi semantici}

A parte i lessemi che concernono la cultura italiana (dož 'doge', karabinjer 'carabiniere', financar 'guardia di finanza (nomen agentis)') e che si usano soprattutto o esclusivamente in riferimento alla realtà italiana, troviamo, tra gli italianismi della lingua slovena, numerosi termini tecnici caratteristici di ambiti d'uso molto eterogenei e relativamente specifici. I campi in cui gli italianismi sono particolarmente frequenti sono i seguenti: musica (es. arija 'aria', sopran 'soprano', maestro 'maestro', kvintet 'quintetto', violina 'violino'), arte (akvarel 'acquerello', štuk 'stucco', terakota 'terracotta', torzo 'torso'), arte della guerra (mušketa 'moschetto', soldat 'soldato'), edilizia (škarpa 'scarpa, scarpata', teraco 'terrazzo'), moda e abbigliamento (damast 'damasco'; kostum 'costume', kostim 'tailleur' - sempre da costume), arredamento (napa 'cappa aspirante', triest. napa, friul. nàpe), commercio, economia e finanza (kapital 'capitale', kredit 'credito', dukat 'ducato', valuta 'valuta'), botanica (oleander 'oleandro'; limona 'limone', špinača 'spinacio', tartuf 'tartufo'), zoologia - particolarmente ittionimi (brancin 'branzino', kalamar 'calamaro', orada 'orata', trilja, in dialetto anche trija e trigla, 'triglia', v. Bezlaj 1959/60: 174) ma anche nomi di altri animali (mula 'mula'; martinček 'lucertola comune', dove si può supporre la base saltamartino con modificazione semantica), gastronomia (brodet 'brodetto', marinirati 'marinare', pica/pizza 'pizza', špageti 'spaghetti', paštašuta 'pastasciutta'), enologia (malvazija 'malvasia', refošk 'refosco'). Registriamo qui anche dei marchionimi, in realtà usati soprattutto con estensione a nomi comuni (jacuzzi/džakuzi 'vasca da bagno con idromassaggio', superga 'scarpetta sportiva', vespa 'motociclo di piccola cilindrata').

Un sottogruppo specifico di lessemi con la radice italiana ha una connotazione peggiorativa; il che non sorprende in quanto spesso lessemi spregiativi vengono importati da ambienti linguistici stranieri. Gli italianismi con valore peggiorativo in 
sloveno si possono riferire sia a difetti fisici ${ }^{1}$ (mutast 'muto', gobast 'gobbo', čotast ven. zotto/zoto 'zoppicante') sia a caratteristiche psicologiche non desiderabili (matast 1. 'sonnolento', 'assonnato', 2. 'matto', 'pazzo'; bimbo 'imbecille'; goljuf 'imbroglione'; mona 'sciocco'; larifari 'ciancia', 'ciarla'; čenča 'ciancia', 'ciarla').

\section{4 Caratteristiche morfosintattiche degli italianismi}

Quanto alla tipologia delle parti del discorso, prevalgono nettamente quelle variabili, tra cui occupano il primo posto i sostantivi, seguiti dai verbi ( $f a l i t i$ 'fallire', tapecirati 'tappezzare') e dagli aggettivi (fin 'fine', siguren 'sicuro', prefrigan 'astuto' con la base derivata da friggere). Tra le parti del discorso invariabili si possono riscontrare avverbi (magari 'magari', solo 'solo'), interiezioni (bravo 'bravo', čiao/čiau/ciao 'ciao') e una congiunzione ( $m a$ ' $m a$ '). L'avverbio solo ha sviluppato anche un uso particolare: può svolgere funzione attributiva, in posizione descrittiva, all'interno di alcune parole composte (solopetje 'canto solistico', soloples 'assolo di danza', soloplesalec/soloplesalka 'primo/a ballerino/a' ecc.). Molto probabilmente anche nella formazione di queste parole si tratta dell'influsso del modello tedesco (cfr. Solosänger(in), Solotanz, Solotänzer(in) ecc.).

Per la maggior parte gli italianismi sloveni sono lessemi integrati. Talvolta il prestito integrato coesiste accanto a quello non integrato (pica/pizza 'pizza', jacuzzi/džakuzi 'jacuzzi'). Tra gli italianismi non integrati si incontrano soprattutto indicazioni di movimenti musicali, quali andante, piano, vivace, e alcuni altri termini dal campo musicale (libretto, accanto a libreto; maestro), mentre in altri casi anche i termini musicali sono stati adattati alle caratteristiche fonomorfologiche della lingua ricevente (fagot 'fagotto', falzet 'falsetto', činele 'piatti' da cinelli attraverso il ted. Tschinelle).

\section{CONCLUSIONE}

Concludendo questa breve e preliminare esposizione degli italianismi in sloveno possiamo osservare che attraverso i secoli l'italiano è stato tra quelle lingue che hanno dato apporti lessicali più significativi alla lingua slovena, accanto al tedesco, francese, croato e, più di recente, l'inglese. Gli italianismi in sloveno riflettono da una parte i settori nei quali l'influsso della cultura italiana è stato particolarmente forte (come nei campi dell'agricoltura, della vita quotidiana, della musica, della gastronomia, dell'arte ecc.) sia a livello più specificamente locale sia a quello europeo; specialmente nel caso degli italianismi «europei» questi ultimi sono spesso pervenuti attraverso la mediazione del tedesco. Allo stesso tempo gli italianismi stanno a testimoniare una strettissima convivenza tra i parlanti delle due lingue rivelando le attività condivise e talvolta una percezione specifica degli sloveni nei confronti dei loro vicini occidentali. Essi narrano, in un modo del tutto originale, una parte della storia di questa convivenza - una storia che, almeno nel campo linguistico, continua a rimanere una ricca miniera.

\footnotetext{
${ }^{1}$ F. Bezlaj (1951: 454) cita ancora puklast 'gobbo' dal ted. puckelig e šantav, šantati 'zoppicante', 'zoppicare' dall'ungheserese santa.
} 


\section{Bibliografia}

I

BSJ

Besedišče slovenskega jezika. [Lessico della lingua slovena]. A cura di Ivanka Šircelj-Žnidaršič. Ljubljana: ZRC SAZU, ${ }^{2}$ 1998. [Prima edizione: a cura di Milena Hajnšek-Holz et al., Ljubljana, ZRC SAZU, 1987, 2 voll.].

\section{ESSJ}

France BezlaJ: Etimološki slovar slovenskega jezika [Dizionario etimologico della lingua slovena]. Ljubljana: Slovenska akademija znanosti in umetnosti/Mladinska knjiga, 1976-2007, 5 voll.

\section{SES}

Marko SNoJ: Slovenski etimološki slovar [Dizionario etimologico sloveno]. Ljubljana: Modrijan, 22003. [Prima edizione: Ljubljana, Mladinska knjiga, 1997].

\section{SSKJ}

Slovar slovenskega knjižnega jezika [Dizionario dello sloveno standard]. Ljubljana: Slovenska akademija znanosti in umetnosti/Državna založba Slovenije, 1980-1991, 5 voll.

\section{II}

BezlaJ, France (1951) «W. Havers, Neuere Literatur zum Sprachtabu.» Slovenski etnograf III-IV, 451-455.

BEZLAJ, France (1959/60) «Nekaj problemov iz ribjih imen.» Jezik in slovstvo V, 170-175.

Оžвот, Martina (2003) «Un caso particolare di italiano e sloveno a contatto: elementi linguistici romanzi nei testi della letteratura slovena di Trieste.»In: Fernando Sánchez Miret (a cura di), Actas del XXIII Congreso Internacional de Linguística y Filología Románica (Salamanca, 24-30 septiembre 2001). Tübingen: Niemeyer, 147-155.

SkUBIC, Mitja (2000) Elementi linguistici romanzi nello sloveno occidentale. Roma: Il Calamo. SNOJ, Marko (2003) «Uvod.» In: id., SES, III-XVI.

ŠEGA, Agata (1998) «Contributo alla conoscenza dei latinismi e romanismi antichi in sloveno.» Linguistica XXXVIII/2, 63-85.

ŠEGA, Agata (2007) «Nekaj ugotovitev o glasovnih značilnostih vulgarnolatinskih predlog za starejše latinizme in romanizme v slovenščini.» Jezikoslovni zapiski [Merkujev zbornik] 13/1-2, 397-408.

ŠEGA, Agata (2008) «Nekaj ugotovitev o razširjenosti starejših latinizmov oziroma romanizmov v slovenščini in drugih slovanskih jezikih.» Keria X/1, 77-87.

ŠTURM, Fran (1927) «Refleksi romanskih palataliziranih konzonantov v slovenskih izposojenkah.» Časopis za slovenski jezik, književnost in zgodovino VI/1-4, 45-85. [Versione italiana: "Riflessi sloveni di consonanti palatali neolatine.» Estratto dal Ce fastu? VIII (1933)/9-10, 30 + VIII pp.]

ŠTuRM, Fran (1928) «Romanska lenizacija medvokaličnih konzonantov in njen pomen za presojo romanskega elementa $\mathrm{v}$ slovenščini.» Casopis za slovenski jezik, književnost in zgodovino VII/1-4, 21-46. 


\section{Povzetek \\ NEKAJ OPAŽANJ O ITALIANIZMIH V SLOVENŠČINI}

$\mathrm{V}$ prispevku so predstavljeni preliminarni rezultati raziskave o italianizmih v slovenščini, katere namen je popis fonda italianizmov in njihova analiza. Izdelan je bil seznam približno 500 italianizmov knjižne oz. standardne slovenščine, vanj pa niso zajeti izrazito narečni italianizmi ozke razširjenosti. Raziskava se je oprla na Slovenski etimološki slovar M. Snoja (2003) kot glavni vir, kot sekundarni viri pa so bila uporabljena še nekatera druga dela.

Ugotovljeno je bilo, da se italianizmi v slovenščini pomembno razlikujejo glede na posrednost ali neposrednost izposoje, glede na pogostnost rabe, prostorsko razširjenost, socialno in funkcijsko zvrstnost, starost, semantična polja, stopnjo prilagojenosti ciljnemu jeziku in nekatera druga merila. Potrjeno je bilo, da je italijanščina eden tistih jezikov, ki so $\mathrm{k}$ slovenskemu besedišču veliko in pomembno prispevali, bodisi z elementi, ki jih je italijanščina dala evropskim jezikom nasploh, bodisi z leksemi, ki so specifični za slovensko prevzemanje iz jezika zahodnih sosedov. Italianizmi v slovenščini pričajo o izjemno tesnem sobivanju slovenskih in italijanskih govorcev skozi stoletja in ponujajo svojevrsten pogled na različne segmente skupne realnosti. Pomenijo nadaljevanje izposojanja od romanskih sosedov, ki se je začelo v najzgodnješi dobi naselitve slovenskih prednikov v vzhodnih Alpah in na Krasu. 